\title{
CO-ADAPTATION AND LOSS OF VARIATION IN TRIBOLIUM
}

\author{
DAVID E. MCCAULEY* \\ Department of Ecology and Evolution, State University of New York at Stony Brook, Stony Brook, \\ New York 11794
}

Received 14.xii.76

\section{SUMMARY}

\begin{abstract}
An increase in development time following the termination of 11 generations of backcrossing of the $b$ allele on to a wild type strain of Tribolium castaneum is ascribed to selection. Co-adaptation of the wild background to the novel locus has altered the fitness relationships among alleles controlling development time and resulted in selection for an increase in this character. The mechanism of increase, conversion of a polymorphism in larval instar number to populations almost entirely monomorphic for the greater number of instars, has apparently reduced the amount of genetic variation present in those loci influencing development time.
\end{abstract}

\section{INTRODUCTION}

THE effect of genetic background upon fitness relationships among alleles is not well understood. Given strong epistatic interactions among loci, differences in alternative genetic backgrounds could alter fitnesses of particular loci. This has been examined experimentally in Tribolium by Sokal and his co-workers (Sokal and Sonleitner, 1968; Sokal and Fujii, 1973; Sokal, Kence and McCauley, 1974). Briefly, the fitness of the black allele $b$, when in competition with its wild type $b^{+}$, is affected to some degree by the genetic background against which the selection occurs.

It seems reasonable to extend this argument to quantitative characters controlled by a number of loci. That is, the fitness of such characters is in part determined by epistatic interactions of these loci with the rest of the genome. Interlocus adjustments of this sort are the mechanistic basis for the concept of genetic co-adaptation (Wright, 1956).

Egg to adult development time in Tribolium castaneum is a readily measured quantitative character that has been shown to have a strong genetic component (Dawson, 1965; Englert and Bell, 1970; McCauley 1974; and others). This is a report on the effects of an alteration in genetic background upon this character.

Genetic strains of Tribolium have characteristically differing development times when measured under ideal (low density) conditions. Typically, the synthetic wild type Purdue Foundation strain (UPF) takes approximately 2 days longer to mature than does the Chicago Black strain (CB) marked with the aforementioned semi-dominant marker $b b$ (Kence, 1973). Presumably the two strains differ at many loci other than this one marker since differences in adult body weight and fecundity have been measured in addition to those in development time (Kence, 1973).

To study the effects of genetic background on the fitness of the $b$ allele, Sokal and Fujii (1973) backcrossed this gene on to the UPF background for

* Present Address: Department of Biology, University of Chicago, Chicago, Illinois 60637.

$39 / 1-\mathrm{K}$ 
up to II generations. By backcross generation eight, development times in the backcross population (BC) and the UPF population were similar both in means and distribution. It appears $b b$ and loci tightly linked to it have no direct effect on development time. After termination of backcrossing several $\mathrm{BC}$ populations were maintained under the same husbandry procedures as both the UPF and CB stocks. Some months later the above authors reassayed their $\mathrm{BC}$ populations and found a small increase in development time which they suggest may indicate a co-adaptive adjustment. The present work was undertaken to examine further changes in development time in the BC populations. Two backcross populations (BC-I, BC-2) had bcen maintained completely isolated from each other as had the UPF and $\mathrm{CB}$ stocks.

\section{Materials AND Methods}

In June 1976 all four populations (UPF, CB, BC-1, and BC-2) were reassayed for development time as follows. Forty grams of medium consisting of 95 per cent sifted whole wheat flour and 5 per cent brewer's yeast were seeded with 200 eggs less than 24 hours old. On day 22 and at 4-day intervals thereafter the culture was sifted and pupae removed. Pupae were kept separately and examined daily for eclosion. Three replicates were run for cach of the four populations at $29 \cdot 5^{\circ} \mathrm{C}$ and 70 pcr cent relative humidity.

\section{Results AND Discussion}

The results are shown in the table. Replicates within populations were similar $(\mathrm{P}>0 \cdot 10$ in all comparisons using Kolmogorov-Smirnov two-sample

TABLE

Menn $(\overline{\mathrm{Y}})$ and variance $\left(\mathrm{s}^{2}\right)$ in number of days required for egg to adult development time in four populations of Tribolium and sample sizes $(\mathrm{n})$ used in estimates

\begin{tabular}{ccccc}
\multicolumn{5}{c}{ Population } \\
\cline { 2 - 5 } & CB & UPF & BC-1 & BC-2 \\
$s^{2}$ & 28.57 & $30 \cdot 44$ & 31.57 & $31 \cdot 74$ \\
$n$ & 538 & 471 & 499 & 466
\end{tabular}

tests) and pooling them allows the calculation of means and distributions of egg to adult development times based on a minimum of 466 individuals per population sampled. These data are illustrated graphically in the fig. As expected, mean development time in UPF was nearly 2 days longer than that of CB. Unexpectedly, mean development time in both BC-I and BC-2 was about I day longer than that of UPF (significant at the 0.01 level) and nearly 3 days longer than that of $\mathrm{CB}$. There was no difference, however, in the means of $\mathrm{BC}-\mathrm{I}$ and $\mathrm{BC}-2$. An examination of the fig. also reveals very littlc difference in the distribution of development times in these two populations.

It appears that selection has acted on development time in the $\mathrm{BC}$ populations during the last 4 years. Since the relationship between UPF and $\mathrm{CB}$ has remained constant, the selection must in some way be a consequence of the introduction of the block of genes containing the $b b$ locus on 
to the wild type background. The process of genetic adjustment by the wild type background to this novel block of genes must have included an alteration of fitness relationships within loci controlling development time. It is of importance to note that both BC-1 and BC-2 populations, isolated for over 5 years, responded so similarly. This reduces the possibility of stochastic processes determining changes in development time.

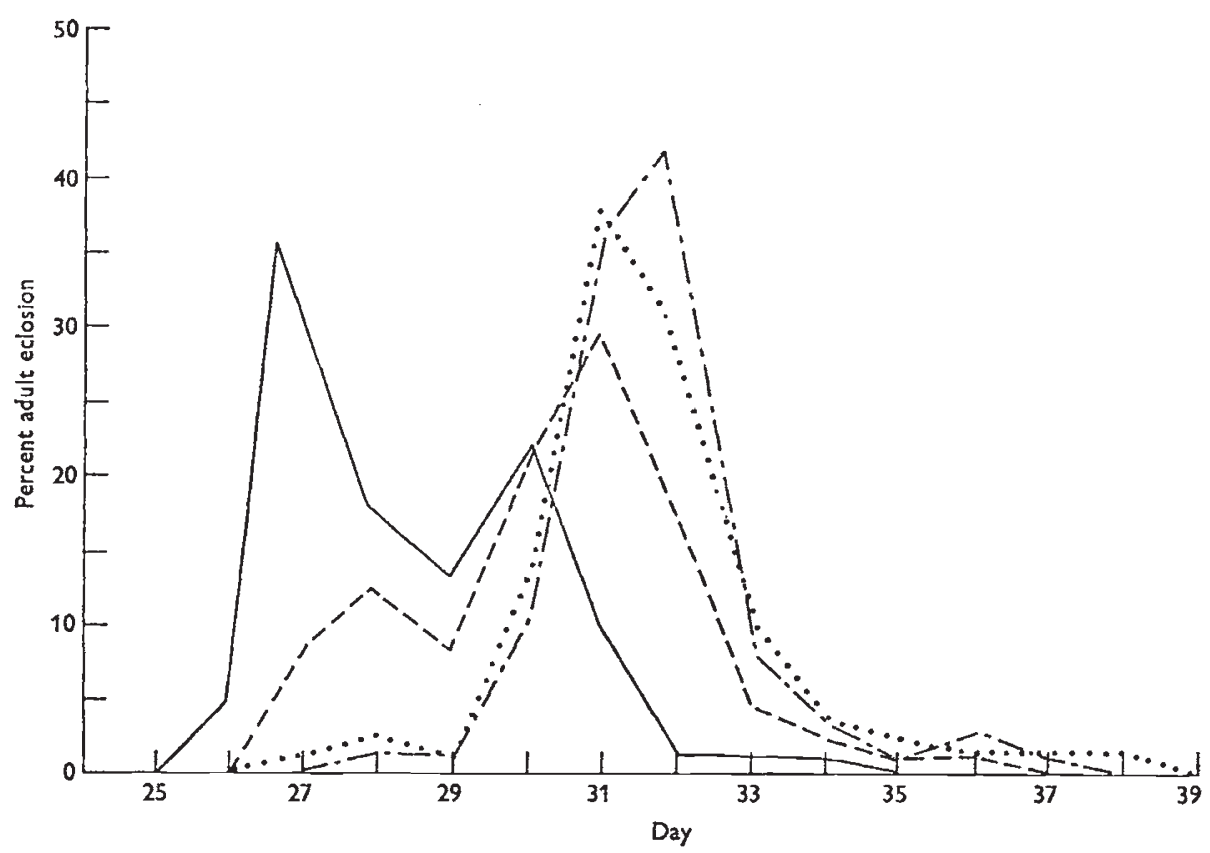

Fig.--Distribution of egg to adult development times in UPF (dashed line), CB (solid line), BC-1 (dotted line), and BC-2 (dots and dashes) populations of Tribolium.

For some reason the presence of the novel genome causes a longer development time to be adaptive. From the fig. it is apparent that both CB and UPF development times are bimodal in their distribution. Kence (1973) has shown that, in UPF at least, this is due to a genetic polymorphism for instar number. His work indicates the trait to be polygenic with the fast development alleles mostly dominant. If this is so, then selection on the BC populations has favoured the extra instar at the expense of the polymorphism. In effect, the co-adaptive process may have resulted in a loss of genetic variation. If Kence's dominance relations hold, then the slow morph would be highly homozygous for all loci controlling development time. It is not unlikely that in complex genetic systems maintainance of genetic polymorphism is to some degree determined by interactions among loci as has been indicated here.

Acknowledgments.-I would like to thank Douglas J. Futuyma, Michael J. Wade, and Robert R. Sokal for their helpful comments concerning this manuscript. This work is contribution number 192 from the Graduate Program in Ecology and Evolution, State University of New York at Stony Brook and was supported by National Science Foundation Grant BMS 6900343A04 to Robert R. Sokal. 


\section{REFERENCES}

DAwSON, P.s. 1965. Estimation of the components of phenotypic variance for development rate in Tribolium. Heredity, 20, 403-417.

ENGLERT, D. C., AND BRLL, A. E. 1970. Selection for time of pupation in Tribolium castaneum. Genetics, 64, 541-552.

KENCE, A. 1973. The effects of variation in larval development on laboratory populations of Tribolizm and houseflies. Ph.D. thesis, State Univ. of N.Y. at Stony Brook, 184 pp. MCCAULEY, D. E. 1974. Selection for changes in developmental period in Tribolium castaneum (Chicago Black strain). Tribolium Information Bulletin, 17, 104-107.

SOKAL, R. R., AND FUJII, K. 1973. The effects of genetic background on the ecology of selection in Tribolium populations. Evolution, 26, 489-512.

SOKAL, R. R., KENCE, A., AND MCGAULEY, D. F. 1974. The survival of mutants at very low frequencies in Tribolium populations. Genetics, 77, 805-818.

SOKAL, R. R., AND SONLEITNER, F. J. 1968. The ecology of selection in hybrid populations of Tribolium castaneum. Ecol. Mongr., 38, 345-479.

WRIGHT, s. 1956. Modes of selection. Amer. Nat., 90, 5-24. 